\title{
Lacunarity and the Size of Road Network as Contributors to Spatial Identity: Informal Patterns VS. Vernacular Urban Settlements
}

\author{
Denada Veizaj", Gjergji Islami, Andrea Maliqari \\ Faculty of Architecture and Urbanism, Polytechnic University of Tirana, Rruga "Muhamet Gjollesha”, Nr. 54, Tirana, Albania
}

Received November 12, 2020; Revised May 28, 2021; Accepted June 15, 2021

\section{Cite This Paper in the following Citation Styles}

(a): [1] Denada Veizaj, Gjergji Islami, Andrea Maliqari, "Lacunarity and the Size of Road Network as Contributors to Spatial Identity: Informal Patterns VS. Vernacular Urban Settlements," Civil Engineering and Architecture, Vol. 9, No. 5, pp. 1283 - 1288, 2021. DOI: 10.13189/cea.2021.090501.

(b): Denada Veizaj, Gjergji Islami, Andrea Maliqari (2021). Lacunarity and the Size of Road Network as Contributors to Spatial Identity: Informal Patterns VS. Vernacular Urban Settlements. Civil Engineering and Architecture, 9(5), 1283 - 1288. DOI: 10.13189/cea.2021.090501.

Copyright $\odot 2021$ by authors, all rights reserved. Authors agree that this article remains permanently open access under the terms of the Creative Commons Attribution License 4.0 International License

\begin{abstract}
This paper aims to contribute to the debate on the "reading" of urban morphologies through quantitative means. The research objective is the establishment of a numeric method for comparing spatial characteristics of different families of urban morphologies, by considering the degree of fragmentation as a key contributor to their spatial identity. The hypothesis elaborated in this paper is that the fragmentation degree of urban textures can be adequately described through a set of fractal parameters. In addition to the fractal dimension, lacunarity is used in order to complete the analytic model. The intricate relations between morphologic characteristics of the built form and urban mobility are analyzed by referring to the network size and network density of the roads system. The experiment consists of two phases: phase one produces binary images of urban samples belonging to different families of urban morphologies. The focus of the experiment is placed on several informal and vernacular zones in Albania. Over the second phase, the values of fractal indexes, the network size and network density on the binary images of urban samples are measured. The outcome of the experiment purports to establish that the spatial characteristics of different urban morphologies can be adequately expressed in numeric terms. Informal urban patterns are characterized by higher values of lacunarity and lower values of roads network densities compared to vernacular ones, even though they appear to have similar values of fractal dimensions. The
\end{abstract}

outcome of the experiment opens up new perspectives in relation to urban design practices and planning processes.

Keywords Lacunarity, Spatial Identity, Road Network Density, Informal Patterns, Vernacular Patterns

\section{Introduction}

The focus on urban morphologies is of a crucial nature for understanding cities and built entities. There is a longstanding preoccupation of academics and professionals for the role that the qualities of the built form have in urban development dynamics [1, 2]. Since the post-modern paradigms, urban facts have been widely seen as complex phenomenon rather than only physical presences [3,4]. On the other hand, the development of digital technologies and techniques, combined with advanced mathematical and geometric concepts brought to researchers and designers new unexpected tools for "reading" and understanding urban facts, by dealing simultaneously with their complexity [5, 6]. In this perspective, the questions raised in the paper are part of the debate that principally focuses in city's architecture and in its reading. More specifically, the current research work aims to theoretically contribute in the following problem: 
How can two samples of urban patterns (as shown in Figure 1) be compared, in terms of their spatial features?

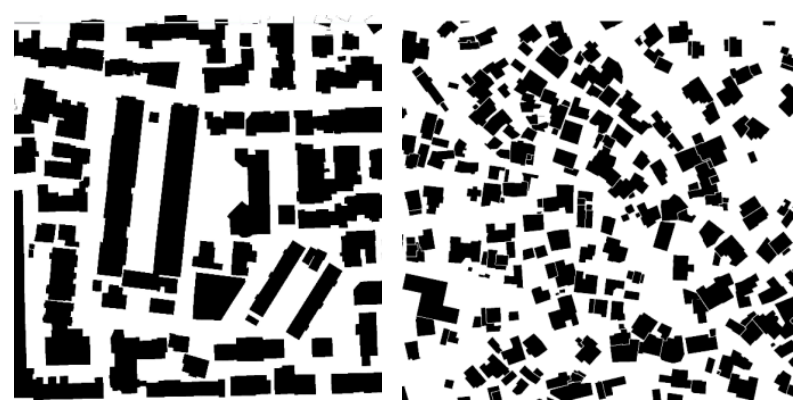

Figure 1. Binary images representing urban samples of different morphogenesis located in Albania: On the left-Urban patterns developed during communism (City of Tirana), on the right-vernacular patterns (City of Gjirokastra). Image produced by the authors

It is obvious, that the paper does not represent the first attempt to deal with such problem [5]. Historically, architects and urban designers have used various tools for highlighting the differences between distinctive urban patterns. It is also crucial to underline that the majority of the tools used are of a descriptive nature. The most common one, is referred to the presence of specific geometric systems.

If the presence of the orthogonal system is perceived in a particular urban texture, the latter is usually labeled as "regular". The opposite happens when the orthogonal system is lacking; urban textures are usually referred as "irregular".

Another widely used method of comparing urban textures is related to the presence or not of pre-design or planning processes (urban morphogenesis) [7]. There are two main families of urban patterns regarding this systemic categorization; self-grown urban patterns, usually addressed as vernacular urban patterns and urban configurations developed under design and planning processes.

The research aims to contribute in the set of theories that refer to quantitative data for depicting spatial features of urban patterns. Notions such as floor area ratio, soil occupancy, or urban density are commonly used for describing spatial characteristics. Still the information appear to be incomplete because the above parameters cannot describe compositional features and qualities.

Can we refer to additional numbers for building a system that properly describes, by simplifying at the same time the process of comparison between different families of urban patterns?

There are significant efforts for using alternative geometric systems in order to read spatial qualities of cities and of urban entities. The first attempts were introduced by M. Batty [8], N. Salingaros [9], S. Salat [10] and P. Frankhausser [11]. They all rely on the concept of additional analytic tools that fractal theory offers by going beyond simple concepts, such as form, geometry and density.
It is important to mention that, the theoretic approach used in this research does not refer to the fractal theory for its concepts of self-similarity, nor even for its metaphorical approach to the city as a fractal organism or entity $[12,13]$. This research uses the fractal indexes for studying the relations between mass and space.

What can fractal geometry offer more than the Euclidian one concerning the process of reading spatial characteristics of the built form? For answering the question, parallel to what Mandelbrot [14] acclaims, an abstract simplified graphic example is proposed, (Figure 2) where Euclidian concepts related to urban density are compared to the fractal ones.

As shown in the image, in the three cases, the same number of black cells is spatially composed in different ways. In the first case, the composition appears highly compact, while in the second and in the third image, its gradual fragmentation offers a more pixelated texture. If we refer to Euclidian concepts, the density (measured as the ratio between the black surface of the cells and the white one) would be the same in the three cases. If we refer to the fractal dimension value, we would obtain very different numbers, the values of which are related to the compactness/fragmentation degree of the composition. The more compact the composition appears, the more the fractal dimension tends to the value of 2 (the absolute planar surface). The more fragmented or pixelated the composition, the more the fractal dimension decreases to the value of 1 [15].

Fractalyse $^{1}$ has been used to apply the calculations of fractal values on binary images [16].

By transposing the problem in the field of urban design, the fractal dimension of two-dimensional representations, would become an important parameter that gives numeric information about the fragmentation degree of urban tissues. This information implies many other interesting urban features, such as scale of space, (by consequence space identity) and urban mobility. Thus, referring to this indicator can result in obtaining significant data in the processes of reading the built form [16].

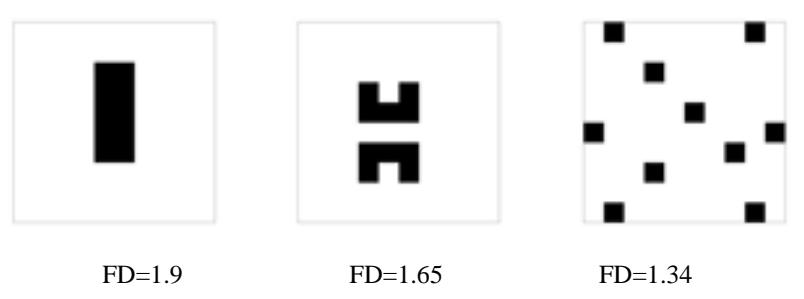

Figure 2. Observing fractal dimension values according to the compactness degree of the composition. Image produced by the authors

1 Software developed by Gilles Vuidel http://www.fractalyse.org/en-home.html 


\section{Methodology: The Theoretic Model}

In order to illustrate how spatial characteristics are expressed in fractal numbers, experimental measurements have been applied in two families of urban patterns, characterized by different morphogenetic processes: Informal urban areas and vernacular urban settlements. Both of the urban samples are located in Albania, as a country characterized by strong urban dynamics during the last three decades. According to the spatial scale of Albanian cities and to the compositional urban entities identified, the opportune size of urban samples lays between the values of $300 \mathrm{~m} \times 300 \mathrm{~m}$ to $500 \mathrm{~m} \times 500 \mathrm{~m}$ (as shown in Figure 3).

The analytic urban cells are extracted from city maps and then converted in binary images through GIS applications. The calculations of fractal dimension values are applied over to 40 samples belonging to vernacular patterns and to informal urban settlements. Because of their similarities in terms of fragmentation degree of built form, the fractal dimension values obtained during the measurements appear to be similar, despite they represent very different cases of spatial features of the built form.

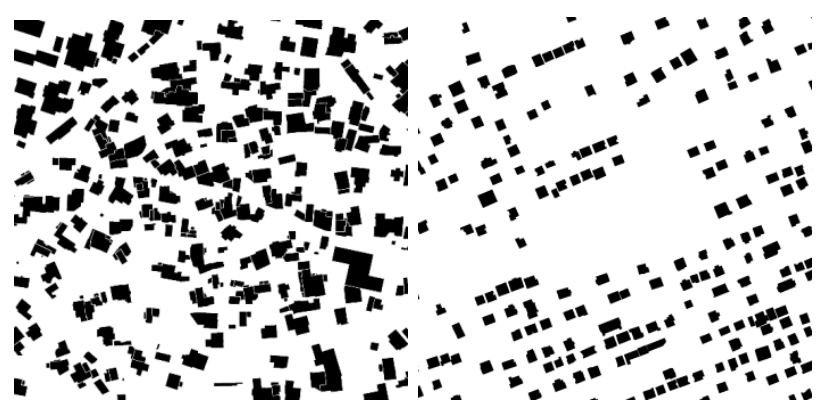

Figure 3. Binary images of two different urban samples. On the left-Vernacular urban texture, on the right-informal urban texture. Image produced by the authors

\subsection{Introducing Lacunarity}

The above paragraph demonstrates how in certain cases urban samples can perform in similar intervals of fractal dimension values, albeit they have different morphogenesis. This fact is related to the alike pixelated degree of their two-dimensional urban texture. In this perspective, additional indexes are necessary for a better description of their spatial characteristics.

In fact, there is a substantial difference between both patterns; vernacular urban patterns are highly fragmented, but homogenous in terms of mass dispersion in space. All the opposite is seen in informal urban patterns. They are as well fragmented spatial compositions, by resulting in highly pixelated textures, but they are at the same time, spatially, very heterogeneous, as long as a large quantity of empty spaces populates the selected samples. Mass (lacunas as well) are not spread uniformly. This fact finds its reflections in the level of urban mobility. A highly fragmented and homogenous spatial structure potentially creates higher number of road nodes, by consequence a larger number of alternatives for connecting two points (Figure 4).
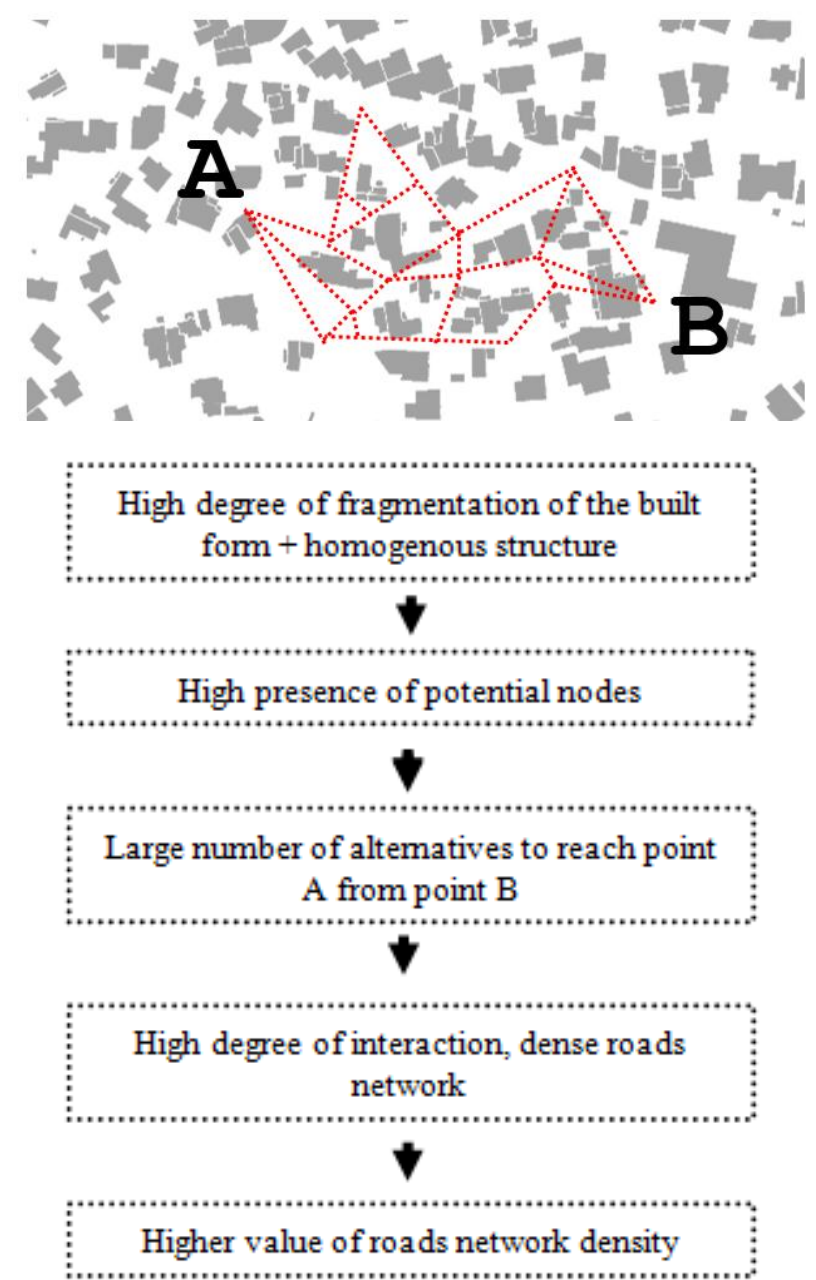

Figure 4. Graph explaining the relations between features of built form (degree of fragmentation) and mobility

Starting by these observations done in real urban conditions, two additional parameters for improving the "numeric description" of urban textures are proposed. The main scope of experimental measurements is to understand relations between fragmentation degree, homogeneity and mobility. For this reason, Lacunarity and road network density are introduced.

$$
U=[F D, \Lambda, k, D]
$$

Where:

U-urban sample characteristics

FD-Fractal Dimension

$\lambda$ - Lacunarity

$k$ - Network size

D-Network density

Since the modern era, the relation between form and function was approached as essential for understanding architecture and urban entities [17]. 
The experiment tends to quantify the interaction between form and mobility. Similar approaches are found in the work and rhetoric produced by A. Sevtsuk [18].

The following paragraphs explain how lacunarity and road network density are measured in the given urban samples.

Lacunarity is an index (part of the fractal theory) that describes the spatial composition of lacunas, by indirectly giving information about the mass behavior [19]. The index gives information about the differences in size of lacunas present in spatial structure. A homogenous spatial structure is characterized by a low value of lacunarity, due to the small difference between the largest lacuna and the smallest one. A heterogeneous spatial structure is characterized by a high value of lacunarity which is translated in a considerable difference between the sizes of lacunas within the structure. For illustrating the concept, we have proposed an example relying another time on abstract binary images [20].

The calculation of the index on the binary images is done by using Fractalyse. The range of lacunarity values lays between 0 and 1000 . See below the increment of the index according to the difference between the sizes of gaps.

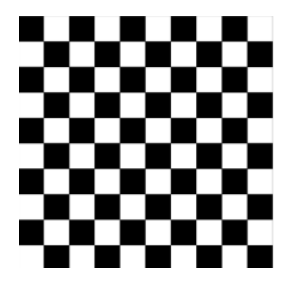

$\lambda=180$

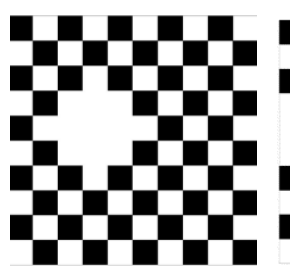

$\lambda=260.3$

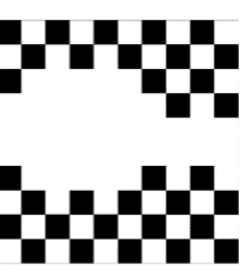

$\lambda=330.3$
Figure 5. Observing/Understanding values of Lacunarity. Experimental measurements are done with Fractalyse. Image produced by the authors

\subsection{Introducing the Size of the Network and Road Network Density}

The last parameter completing the theoretic approach proposed in the paper is the road network size and density.

According to network theory, [21] the network density depends by two factors: the number of nodes present in a network, and how well connected they are among each-other. For each network composed by $k$ nodes, the maximal number of connections is calculated by the formula:

$$
L(m)=\frac{k *(k-1)}{2}
$$
$k$.

The size of a network is given by the number of nodes

The density of a network of $k$ nodes is given by the ratio between the actual or real number of links and the maximal one.

$$
D=\frac{l(r)}{l(m)}
$$

\subsection{The Design of the Experiment}

The experiment consists in measuring the three presented indexes in different urban samples.

The first step requires producing urban samples from GIS information. As shown in Figure 6, the binary images of 30 samples extracted from Tirana and Gjirokastra (both cities located in Albania) are produced and used for calculations of the fractal dimension and lacunarity.

The second step requires the identification of the road network in the chosen samples, and then the calculation of its density.
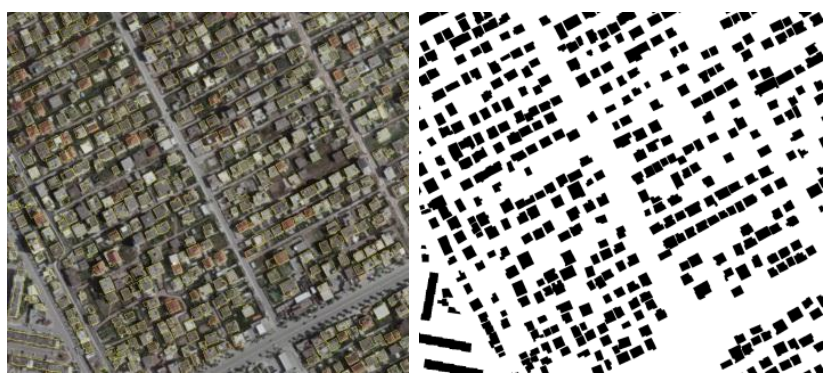

Figure 6. Generating binary images from urban samples and maps. Image produced by the authors

Figure 7, offers a graphic illustration of the methodology used for measuring the road network density in urban patterns. The road system is converted in a matrix of points (horizontally and vertically), representing the nodes of the network. Cells composed by connected nodes are valued with 1 , and non-connected ones with 0 . By counting the cells containing the number 1 , and then divide the number by two, we obtain the real links of the network. The maximal number of links is calculated by the formula (1.2), and therefore the density of the network (1.3) results as the ratio between the both values. 


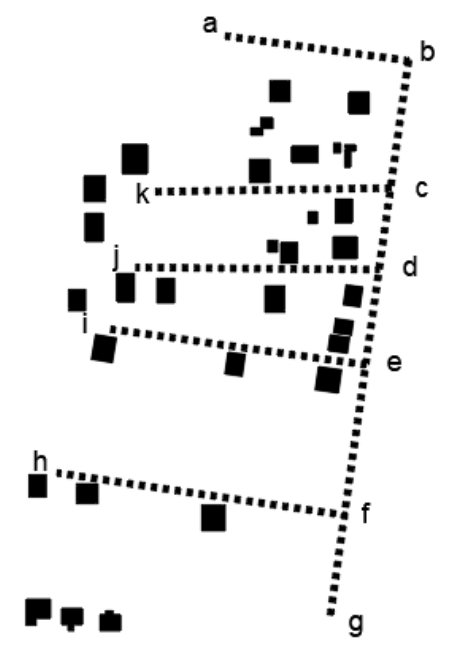

\begin{tabular}{|c|c|c|c|c|c|c|c|c|c|c|c|}
\hline & $a$ & b & C & d & $\mathbf{e}$ & $f$ & $\mathbf{g}$ & $\mathbf{h}$ & $\mathbf{i}$ & $\mathbf{j}$ & $k$ \\
\hline $\mathbf{a}$ & & 1 & & & & & & & & & \\
\hline b & 1 & & 1 & & & & & & & & \\
\hline C & & 1 & & 1 & & & & & & & 1 \\
\hline d & & & 1 & & 1 & & & & & 1 & \\
\hline e & & & & 1 & & 1 & & & 1 & & \\
\hline$f$ & & & & & 1 & & 1 & 1 & & & \\
\hline g & & & & & & 1 & & & & & \\
\hline h & & & & & & 1 & & & & & \\
\hline i & & & & & 1 & & & & & & \\
\hline j & & & & 1 & & & & & & & \\
\hline k & & & 1 & & & & & & & & \\
\hline
\end{tabular}

$\mathrm{k}=11, \mathrm{~d}=0.18$

Figure 7. Converting the road system into a matrix of binary values. Image produced by the authors

Table 1. Outcome of experimental findings

\begin{tabular}{|c|c|c|c|c|c|}
\hline & $\begin{array}{c}\text { Fractal Dimension } \\
(1-2)\end{array}$ & Lacunarity (0-1000) & $\begin{array}{c}\text { Dendricity } \\
(1-2)\end{array}$ & $\begin{array}{c}\text { Network Size } \\
\text { (for the same } \\
\text { size of samples) }\end{array}$ & $\begin{array}{c}\text { Road Network Density } \\
\text { [for the same size of } \\
\text { network, (9 } \leq \mathrm{k} \leq 23)]\end{array}$ \\
\hline $\begin{array}{c}\text { Vernacular Urban } \\
\text { Patterns (300m x 300m) }\end{array}$ & $1.511-1.737$ & $288-335$ & $1.426-1.457$ & $13-23$ & $0.33 \leq \mathrm{d} \leq 0.138$ \\
\hline $\begin{array}{c}\text { Informal Urban Patterns } \\
(300 \mathrm{~m} \times 300 \mathrm{~m})\end{array}$ & $1.301-1.649$ & $270-479$ & $1.15-1.41$ & $9-13$ & $0.25 \leq \mathrm{d} \leq 0.08$ \\
\hline
\end{tabular}

The summary of all the data extracted from the experimental measurements are expressed in Table 1.

\section{Results and Discussion}

By observing the experimental outcomes, the first important statement is it that in both cases are obtained similar values of the fractal dimension.

In vernacular patterns, the fractal dimension values result within the intervals of 1.511 to 1.737 , and in the case of informal settlements, the fractal dimension values are between 1.301 and 1.649.

In these cases, the spatial differences of urban textures are expressed by the lacunarity values.

In vernacular urban patterns, lacunarity lays in the range of 288 to 335 . In informal urban settlements lacunarity values appear in between the interval of 270 to 479. Referring to the outcomes, it is reasonable to admit that vernacular urban patterns are highly fragmented and the free space is distributed homogeneously. The opposite happens in the case of informal settlements. They also appear highly fragmented, but the presence of lacunas of different sizes gives them heterogeneous spatial qualities.

Urban mobility as well is affected by fractal dimension values and lacunarity.

High fractal dimension values combined with low values of lacunarity result in high values of the size of the road network. This combination of parameters describes spatially in a proper manner vernacular urban patterns.

High fractal dimension values combined with high values of lacunarity result in low values of road network size. This combination of parameters describes spatially in a proper manner informal urban patterns.

At the same time, if we compare two urban samples, characterized by the same size of road network, the density appears higher in the case of vernacular urban patterns. This because of the higher number of actual links between nodes.

\section{Conclusions}

The research outcome purports to demonstrate that the degree of fragmentation of urban texture is a key element in perceiving and discussing urban identity. The fractal theory, if combined with network concepts can become a powerful tool for describing spatial characteristics of urban morphologies. A better understanding of relations between urban form and mobility can give important insights into urban functions [22].

In a more practical level, the outcome of the research can be useful for building the catalogue of urban patterns that compose the physical layout of the city. It might aid planning and policy-making processes, e.g., by manipulating certain parameters, it is possible to improve urban qualities in present urban contexts. As a way of an example, for improving urban mobility in informal 
settlements, a decrease the lacunarity index might be necessary. This statement can be translated in a series of urban actions that reduce the presence of urban voids and improve linkages between the nodes of the roads network.

The outcome of this research can be of use in the design process of new urban areas. In order to provide for the continuity in terms of urban and spatial identity between the old zones and the new ones, maintaining a similar degree of fragmentation can turn out useful in their spatial aggregation. In terms of planning instruments, the fractal dimension, lacunarity and road network size and density can be used in addition to common technical parameters such as floor area ratio or soil occupancy coefficient, in order to assure a better grasp of urban development.

\section{Acknowledgements}

We are very grateful to GIS experts for their constructive suggestions in improving the experimental measurements.

\section{REFERENCES}

[1] C. Portzamparc and P. Sollers. Writing and Seeing Architecture, Press 2000.

[2] A. Sevtsuk. Networks of the built envirenment, Birkhauser, 2013.

[3] N. Ellin. Post-modern Urbanism, Princeton Architectural Press, 1999.

[4] A. Rossi. The Architecture of the City, MIT Press 1984.

[5] C. Alexander. The City is not a Tree. Architectural Forum, 122(No. 1), pp. 58-62, 1965.

[6] M. Batty. Cities and Complexity, Cambridge, MIT Press. 2005.

[7] N. Salingaros. Computional Urbanism. Complexity theories of cities have come of age. Berlin, Springer, pp. 245-268.
2012.

[8] M. Batty. Fractal Cities, Oxford Press. 1994.

[9] N. Salingaros. Design for a Living Planet. Settlement, Science and the Future Paperback. Sustasis Press, 2015.

[10] S. Salat. Fractal cities. A geometry of function and form. Hermann, 2012.

[11] P. Frankhauser. Fractal geometry for measuring and modelling urban patterns. The Dynamics of Complex Urban Systems. Physica Verlag, pp. 241-243, 2008.

[12] S. Salat. Fractal cities. A geometry of function and form. Hermann, 2012.

[13] N. Salingaros. Twelve lectures on architecture Alghoritmic sustainable design. Sustasis Press, 2010.

[14] B. Mandelbrot. Fractal Geometry of Nature. W.H. Freeman and Company. New York, 1983.

[15] P. Frankhauser. Simulation fractale d'urbanisation MUP-city, un modèle multi-échelle pour localiser de nouvelles implantations résidentielles. Revue internationale de geomatique, 20(3), pp. 303-329, 2010.

[16] Frankhauser, P. Comparing the fractality of European urban neighbourhoods: do national contexts matter? Journal of Geographic System, pp. 1-20, 2010.

[17] A. Sevtsuk, How we shape our cities and then they shape us. MAJA the Estonian Architectural Review, 2(72), pp. 10-15, 2012.

[18] A. Sevtsuk. Path and Place: A study of Urban Geometry and Retail Activity in Cambridge and Somerville, MA, Massachusetts Institute of Technology, PhD dissertation in Urban Design and Planning, 2010.

[19] A. Cloitre. Characterizing the lacunarity of random and deterministic fractal sets. Physical Review, 44(6), pp. 3552-3558, 1991.

[20] P. Dong. Lacunarity for spatial heterogeneity measurement in GIS. Geographic Information Sciences, 6(1), 20-26, 2000.

[21] MIT. Lectures on Networks, Cambridge: MIT Press, 2012.

[22] A. Sevtsuk. Analysis and Planning of Urban Networks. J. Rokne, Encyclopedia of Social Network Analysis and Mining. sSpringer, pp. 24-37, 2014. 